\title{
COVID-19 and Quarantine: Expanding Understanding of How to Stay Physically Active at Home
}

\author{
Alberto Souza Sá Filho ${ }^{1,2 *}$, Thiago Gottgtroy Miranda ${ }^{1}$, Carolina Cavalcante de Paula ${ }^{1,3}$, \\ Silvio Roberto Barsanulfo ${ }^{1}$, Diogo Teixeira ${ }^{4,5}$, Diogo Monteiro ${ }^{6,7}$, Luis Cid ${ }^{7,8}$, \\ Claudio Imperatori ${ }^{5,9}$, Tetsuya Yamamoto ${ }^{5,10}$, Eric Murillo-Rodriguez ${ }^{5,11}$, \\ Sandra Amatriain Fernández ${ }^{12}$, Henning Budde ${ }^{5,13}$ and Sergio Machado ${ }^{5,14,15}$
}

${ }^{1}$ Post Graduate Program of University Center of Anápolis (UniEVANGÉLICA), Anápolis, Brazil, ${ }^{2}$ Physical Education Department of Universidade Paulista (UNIP-Campus Flamboyant), Goiânia, Brazil, ${ }^{3}$ Department of Cell and Developmental Biology, Institute of Biomedical Sciences (ICB), University of São Paulo (USP), São Paulo, Brazil, ${ }^{4}$ Faculty of Physical Education and Sport, Universidade Lusófona de Humanidades e Tecnologias (ULHT), Lisbon, Portugal, ${ }^{5}$ Intercontinental Neuroscience Research Group, Mérida, Mexico, ${ }^{6}$ Department of Human Kinetics, ESECS, Polytechnic of Leiria, Leiria, Portugal, ${ }^{7}$ Research Center in Sport, Health and Human Development (CIDESD), Vila Real, Portugal, ${ }^{8}$ Sport Science School of Rio Maior (ESDRM-IPSantarém), Rio Maior, Portugal, ${ }^{9}$ Cognitive and Clinical Psychology Laboratory, Department of Human Science, European University of Rome, Rome, Italy, ${ }^{10}$ Graduate School of Technology, Industrial and Social Sciences, Tokushima University, Tokushima, Japan, "11 Escuela de Medicina, División Ciencias de la Salud, Universidad Anáhuac Mayab, Yucatán, Mexico, ${ }^{12}$ Faculty of Sport Sciences and Physical Education, University of A Coruña, A Coruña, Spain, ${ }^{13}$ Faculty of Human Sciences, Medical School Hamburg, Hamburg, Germany, ${ }^{14}$ Laboratory of Physical Activity Neuroscience, Physical Activity Sciences Postgraduate Program of Salgado de Oliveira University (PPGCAF/UNIVERSO), Niterói, Brazil, ${ }^{15}$ Laboratory of Physical Activity Neuroscience, Neurodiversity Institute, Queimados, Brazil

Keywords: COVID-19, HIIT (high intensity interval training), aerobic exercise, Tabata protocol, sedentarism

\section{CURRENT SCENARIO}

The coronavirus disease 2019 (COVID-19) is today the biggest public health challenge in the world (Park, 2020). The first case of COVID-19 was diagnosed on December 8, 2019, in Hubei province, China. From that day, in just over 3 months, the virus has spread to more than 177 countries/areas/territories around the world, with more than 266,073 confirmed cases and 11,184 deaths, according to WHO on March 21, 2020 (WHO, 2020). The most common clinical manifestations of COVID-19 are mild flu-like illness, potentially lethal acute respiratory distress syndrome, or fulminant pneumonia. As a result, numerous countries have decided to implement (some by government decrees, as well as martial laws) the establishment of mandatory social distance in a family environment, closing non-essential commercial environments, in an attempt to reduce the peak of the infection curve (Lewnard and Lo, 2020).

We know that a large part of the world population is far from the minimum conditions of physical exercise recommended by the American College of Sports Medicine (ACSM) to improve the health component (Katzmarzyk et al., 2019). This fact would give important relevance to the level of physical activity exercised by the population throughout the day. However, once the extreme hypokinetic behavior is implemented as a result of the quarantine, a cycle of perverse events begins, making part of the population more vulnerable to the deleterious effects of acute and chronic diseases, including respiratory tract infections (Hall et al., 2020).

In a recent position paper presented by Chen et al. (2020), the authors try to propose to the general population to continue exercising regardless of the current moment the world is living. In fact, as the authors mention, based on other researchers, "anything is better than nothing," and the sedentary lifestyle is something that should not be encouraged, i.e., any energy expenditure added to the routine of these people would be significant. Recommendations for the population to keep regularly active highlight only a minimum applicable technical basis, without presenting any 
TABLE 1 | Recommendations for exercising at home.

\begin{tabular}{|c|c|c|}
\hline & Aerobic exercises & Strength exercises \\
\hline Conception & Prolonged or short term exercises using large muscle groups & $\begin{array}{l}\text { Localized exercise with body weight, or free weight } \\
\text { Exercises (include all major muscle) }\end{array}$ \\
\hline Configuration & \multicolumn{2}{|l|}{ Merging one or more strength component with an aerobic component (see Table 2) } \\
\hline Frequency & \multicolumn{2}{|l|}{ 3-5 days/week (consecutive days for high levels of fitness) } \\
\hline Time & \multicolumn{2}{|l|}{ 10-30 min a day. This can be accumulated continuously or in shorter 10-min blocks } \\
\hline Intensity & \multicolumn{2}{|l|}{$\begin{array}{l}\text { Moderate effort ( } 40-59 \% \text { of heart rate-HR) for long workouts (RPE 3-4) or } \\
\text { for lower levels of conditioning; } \\
\text { Moderate to high effort }(60-85 \% \text { of HR)-for intermediate workout times } \\
\text { (RPE 4-6); } \\
\text { High effort ( }>85 \% \text { of HR) - for short workouts (RPE }>7 \text { ) }\end{array}$} \\
\hline Volume & \multicolumn{2}{|c|}{150 or more min/week are required. $4-6$ sets of $6-20$ repetitions for selected exercise. $3-5$ exercises for workout } \\
\hline Workout form & \multicolumn{2}{|c|}{ (a) Mobility and warm up (5-10 min); (b) core or strength (5-10 min); (c) multimodal workout (5-20 min) } \\
\hline
\end{tabular}

TABLE 2 | Proposal of exercises for workouts configuration.

\begin{tabular}{ll}
\hline Upper body & Lower body \\
\hline Push up (or adapted) & Hip trust (on the ground) \\
Pull ups (or adapted) & Squat or split squat \\
Handstand push up (or inverse & Sumo squat \\
press on chair) & \\
Ball throw (or adapted) & Lunge (or walking lunge) \\
Dips on chair or box & Pistol (advanced) \\
$\begin{array}{l}\text { Shoulder push up (on the } \\
\text { ground) }\end{array}$ & Good morning \\
Adapted bent over row (pulling a & \\
towel) & Adapted deadlift or single leg \\
\hline Aerobic demand & deadlift \\
\hline Jumping jacks & Core \\
Jump rope (single or double & Hollow body (or hold) \\
under) & Arch body (or hold) \\
Burpees & \\
Box jump (stairs jump) & Sit ups \\
Box jump over (on chair) & Plank or side plank \\
Skipping (performed in a hallway) & Turkish get up \\
Sprawl & Russian twist \\
\hline
\end{tabular}

suitable parameters for carrying them out. From the initial positioning of Chen et al. (2020), the ACSM via publication on the website of the journal Medicine \& Science in Sports \& Exercise (ACSM, 2020; WHO, 2020), as well as other institutions (ACSM, 2020; WHO, 2020), expanded the proposal about the practice of physical exercise to be performed at home. Tasks such as brisk walking, up and down stairs, dance, jump rope, yoga exercises, and bodyweight strength training are also recommended for indoor workout (Table 1).

\section{EXPANDING RECOMMENDATIONS FOR PRACTICING EXERCISE AT HOME}

The suggestions proposed by the ACSM (2020), WHO (2020), although more consistent, still do not offer a concrete logic to be applied and controlled. Moreover, the statement "Some activity is better than none" makes more sense when we talk about people practicing any physical activity at a beginner level, therefore merely minimally physically active (Chen et al., 2020). Thus, for practitioners of physical exercise at levels that require moderate to high metabolic and strength demand, or even athletes, these would be susceptible to detraining. According to the basic premises related to training, an ideal stimulus must be administered for the adaptation to occur properly, and that condition may not be prioritized with such positions.

Considering the theoretical rationale prevalent in the literature, we believe that the suggestions proposed can be expanded in order to integrate groups of individuals who have moderate to high physical conditioning and not only sedentary individuals, providing better guidance on how to proceed during the quarantine period and offering the possibility of followup training even after the quarantine has ended. For such, the conviction that traditional strategies of aerobic endurance exercise may not be a suitable strategy for application in the residential environment (understanding that majority of the population does not have stationary bikes, arm or rowing ergometers at home), so the interval exercise pattern should be primarily stimulated, with or without the use of any viable resource of overload implementation. In combination with this proposal, the concomitant increase in intensity (vigorous to high intensity) is also essential to promote adaptive results independently of the initial fitness level.

The effects of high-intensity interval training (HIIT) are well-established in the literature for healthy people (Gormley et al., 2008) and those with some comorbidity (obesity, heart disease, diabetics) (Ballesta Garcia et al., 2019; Taylor et al., 2019). More recently, the effects of HIIT have been presented in sedentary individuals, suggesting that the application of interval exercises would be viable, consolidating itself as an important strategy for health promotion (Dorneles et al., 2019; Reljic et al., 2019). The literature shows significant physiological responses derived from different types of interval protocols (Paoli et al., 2012; Buckley et al., 2015; Box et al., 2019), and the improvement in performance seems to be related to the physiological mechanism of inducing mitochondrial biogenesis from the expression of the PGC-1 alpha transcription coactivator, as well as catalyzing enzymes of both the glycolytic 
TABLE 3 | Examples of training session configurations based on intensity control from internal load.

\begin{tabular}{|c|c|c|c|c|c|}
\hline \multicolumn{3}{|c|}{ Lower body program } & \multicolumn{3}{|c|}{ Upper body program } \\
\hline \multirow[t]{2}{*}{2 sets } & \multicolumn{2}{|c|}{ Specific mobility (hip) } & 2 sets & \multicolumn{2}{|c|}{ Specific mobility (shoulders) } \\
\hline & $50 x$ & Jump rope & & $50 x$ & Skipping \\
\hline Core & (10 min) & RPE 4-6 & Strength & (10 min) & $\mathrm{RPE}>7$ \\
\hline Workout & (15 min) & RPE 4-6 & Workout & (15 min) & $\mathrm{RPE}>7$ \\
\hline \multirow[t]{4}{*}{ AMRAP } & $20 x$ & Walking lunges & MAX RFT & $20 x$ & Push up \\
\hline & $20 x$ & Sprawl & & $20 x$ & Dip on box \\
\hline & $20 x$ & Split squat & & $20 x$ & Mountain climbers \\
\hline & $50 x$ & Double under & & $20 x$ & Burpees \\
\hline
\end{tabular}

and oxidative systems (Gibala et al., 2006; Gibala, 2009). Such adaptations promote greater efficiency metabolic rate in energy production and buffering capacity.

In a new perspective, evidence indicates that HIIT performed with body weight can promote significant adjustments in strength, hypertrophy (Kikuchi and Nakazato, 2017), and the cardiorespiratory system. For instance, the Tabata protocol would fit as an interesting tool to be performed at home (Tabata et al., 1996). Basically, it consists of performing stimulus $8 \times 20 \mathrm{~s}$ interspersed with 10 -s recovery, a total of $4 \mathrm{~min}$. The protocol is still performed more than once during an exercise session and with different exercise compositions. Emberts et al. (2013) reported mean values of $74 \%$ of $\mathrm{VO}_{2 \max }$ [rate perceived exertion (RPE) averaged $15.4 \pm 1.3$ ] and $86 \%$ of $\mathrm{HR}_{\max }(156 \pm 13 \mathrm{bpm})$ during two types of Tabata workouts (e.g., mountain climbers, push-ups, split squat, box jumps, burpees, squats, lunges, Russian twist). This level of workout is a sufficient stimulus to generate adaptations to the cardiorespiratory component, and these data are superior to the recommendations proposed by the ACSM. Moreover, the increase in intensity seems to be the key to maintain the gains obtained before COVID-19 (Hickson et al., 1985).

To better target the perspective postulated here in our article, as well as to better interpret the designs positioned in Table 2, Buckley et al. (2015) proposed a high-intensity multimodal training format as a way to reduce the time required for multiple adaptations. For this, the authors compared the physiological responses of the traditional HIIT performed in a rowing ergometer versus multimodal training, involving analysis of different manifestations of strength, in addition to maximum aerobic power and anaerobic capacity. Thirty-two recreational trained participants performed $60 \mathrm{~s}$ "all out" and a 3-min recovery (total of 4 min per series). The multimodal HIIT protocol was configured as follows: a strength exercise for 4-6 repetitions, an accessory movement for 8-10 repetitions, and a metabolic component conducted all out for the remainder of the $60 \mathrm{~s}$. The results were significantly promising, resulting in similar responses in aerobic and anaerobic performance tests; however, multimodal HIIT showed significant improvement in all parameters of different manifestations of strength.

It is suggested, therefore, that the configuration of multimodal workouts be constructed in a similar way to that reported in the literature, and the control of exercise overload (internal load) would be performed based on the RPE ( $0-10$ in combination with session time (Foster et al., 2001). Table 2 shows a coherent exercise division format, and Table 3 shows examples of training session configurations.

\section{CAN EXERCISE INTENSITY COMPROMISE THE IMMUNE SYSTEM?}

Finally, establishing the relationship between the stresses generated from physical exercise at home and the immune system is an important point to be considered during this quarantine period (Amatriain-Fernandez et al., 2020a,b). Nieman (2007) proposes an open window of alteration of the immune system after physical exercise, and such manifestation would occur with significant magnitude in the face of long-lasting endurance, such as in a marathon, or also in the face of extremely heavy efforts. However, little is known about the immune responses to short interval exercise, but current evidence suggests that HIIT seems to be beneficial for the immune system (Bartlett et al., 2017, 2018; Born et al., 2017; Durrer et al., 2017; Dorneles et al., 2019; Steckling et al., 2019; Khammassi et al., 2020), although evidence still points to a higher increase in the percentage of leukocytosis after HIIT exercise (Jamurtas et al., 2018).

So, Bartlett et al. (2017) investigated in 27 sedentary adult individuals the potential of immune response induced by continuous aerobic training of moderate intensity (MICT) and HIIT (volume 57\% smaller). After 10 weeks, there was a significant improvement in the capacity of bacterial phagocytosis by neutrophils ( +16 vs. $+15 \%$, respectively, for HIIT and MICT) and monocytes (14 vs. 19\%, respectively, for HIIT and MICT) for both training groups. Also with a more recent perspective, Born et al. (2017) demonstrated that HIIT, in addition to the superior adaptive responses on the ability to perform exercise (time to 
TABLE 4 | Main positive and negative results from the perspective of HIIT and the changes resulting from this training model.
Durrer et al. (2017)
Objective:
To determine the impact of a single session of HIIT on cellular, molecular, and circulating markers of inflammation in individuals with Type 2 Diabetes (T2D)
Participants: $\quad$ Participants with T2D $(n=10)$ and healthy $(\mathrm{HC})$ age-matched controls $(\mathrm{HC} ; n=9)$
Intervention:
Measures:
Acute bout of HIIT $(7 \times 1$-min at $85 \%$ maximal aerobic power output), separated by 1-min recovery on a cycle ergometer
Outcome:
Blood samples Pre, Post, and 1-h Post. Inflammatory markers on leukocytes and tumor necrosis factor (TNF)- $\alpha$
Outcome:
(a) significantly $\downarrow$ levels of toll-like receptor (TLR); expression on both classical and CD16 ${ }^{+}$monocytes assessed at Post and 1-h Post compared with Pre; (b) significantly $\downarrow$ LPS-stimulated TNF- $\alpha$ release in cultures at 1 -h Post; (c) significantly lower levels of plasma TNF- $\alpha$ at 1 -h Post. There were no differences between T2D and HC except for a larger decrease in plasma TNF- $\alpha$ in HC vs. T2D

Bartlett et al. (2018)

Objective:

Participants:

Intervention:

Measures:

Determine whether 10 weeks of a walking-based HIIT program would be associated with health improvements. Assess whether HIIT was associated with improved immune function, specifically antimicrobial/bacterial functions of neutrophils and monocytes

Twelve physically inactive adults

$3 \times 30$-min sessions/week of $10 \geq 60$-s intervals of high intensity (80-90\% $\mathrm{VO}_{2 \text { reserve }}$ ), and rest of $50-60 \% \mathrm{VO}_{2 \text { reserve }}$

Pre- and post-aerobic and physical function; self-perceived health; C-reactive protein (CRP), and erythrocyte sedimentation rate (ESR); plasma interleukin (IL)-1 $\beta$, IL-6, chemokine (C-X-C motif) ligand (CXCL)-8, IL-10, and tumor necrosis factor (TNF)- $\alpha$ concentrations; and neutrophil and monocyte phenotypes and functions

Outcome:

$\mathrm{VO}_{2 \max } \uparrow 9 \%$; Neutrophil migration toward CXCL-8, phagocytosis of Escherichia coli, and ROS production all increased following training. The frequency of differentiation 14-positive $\left(\mathrm{CD} 14^{+}\right) / \mathrm{CD} 16^{+}$monocytes was reduced, with both non-classical (CD14 dim/CD16 $\left.{ }^{\text {bright }}\right)$ and intermediate (CD14 ${ }^{\text {bright }} / C D 16^{\text {positive }}$ ) monocytes being reduced; Expression of Toll-like receptor 2 (TLR2), TLR4, and HLA-DR was reduced and monocyte phagocytosis of E. coli increased

Bartlett et al. (2017)

Objective:

Participants:

Intervention:

Measures:

Compared the impact of HIIT and moderate-intensity continuous training (MICT) on immune function in sedentary adults

Twenty-seven healthy sedentary adults

HIIT (>90\% maximum heart rate) or MICT (70\% maximum heart rate) group training program

$\mathrm{VO}_{2 \text { peak }}$, neutrophil and monocyte bacterial phagocytosis and oxidative burst, cell surface receptor expression, and systemic inflammation were measured before and after the training

Outcome:

Total exercise time was $57 \%$ less for HIIT; Significantly improved $\mathrm{VO}_{2 \text { peak }}$ for both; Oxidative burst and monocyte phagocytosis and percentage of monocytes producing an oxidative burst were $\uparrow$ by training similarly; Expression of monocyte but not neutrophil CD16, TLR2, and TLR4 was $\downarrow$ by training similarly in both groups; No differences in systemic inflammation were observed for training

Khammassi et al.

(2020)

Objective:

Compare the effects of HIIT and moderate-intensity continuous training (MCT) on hematological biomarkers in active young men (9 weeks/3 training per week)

Participants: $\quad$ Sixteen men aged 18-20 years were randomly assigned to HIIT or MCT group

Intervention:

HIIT: (30 s at 100\% of maximum aerobic velocity/30 s rest at 50\%); MCT sessions were matched for workload based on the total distance in HIIT

Measures: $\quad V_{2} \quad \quad$ max red blood cell, hemoglobin, hematocrit, mean corpuscular volume, mean corpuscular hemoglobin, mean corpuscular hemoglobin concentration, leukocyte, neutrophil, lymphocyte, monocyte, and eosinophil count

Outcome:

No significant change was observed in maximal aerobic velocity and estimated $\mathrm{VO}_{2}$ max in both groups; Leukocyte, lymphocyte, neutrophil, and monocyte count showed significant improvements in response to the MCT; The MCT intervention favored an increase in the number of immune cells

Jamurtas et al. (2018)

Objective:

Evaluated the effects of HIIT on hematological profile and redox status compared with those following traditional continuous aerobic exercise (CET)

Participants:

Twelve healthy young men participated in a randomized crossover design under HIIT and CET

Intervention:

Measures:

HIIT: $4 \times 30$-s sprints on a cycle-ergometer/4 min of recovery. CET: 30-min cycling on a cycle ergometer at $70 \%$ of their $\mathrm{VO}_{2} \mathrm{max}$

Blood was measured at baseline, immediately after, 24, 48, and $72 \mathrm{~h}$ post-exercise and was analyzed for complete blood count and redox status (thiobarbituric acid reactive substances, [TBARS]; protein carbonyls, [PC]; antioxidant capacity total, [TAC]; catalase and uric acid) White cells $\uparrow$ immediately post-exercise (HIIT: 50\% and CET: $31 \%$, respectively); HIIT $\uparrow+22 \%$ PC post-exercise compared to CET; HIIT $\uparrow$ $+16 \%$ TAC immediately post-exercise and at $24 \mathrm{~h}$ post-exercise (11\%), while CET $\uparrow$ TAC only post-exercise (12\%, $p<0.05)$; Both HIIT and CET $\uparrow$ uric acid immediately post- ( 21 and 5\%, respectively) and $24 \mathrm{~h}$ ( 27 and 5\%, respectively); There were no significant changes for TBARS and catalase following either exercise protocol

Born et al. (2017)

Objective:

Evaluate the mucosal immune function and circadian variation of salivary cortisol, Immunoglobin-A (slgA) secretion rate and mood during a period of high-intensity interval training (HIIT) compared to long-slow distance training (LSD)

Participants: $\quad 28$ Recreational male runners

Intervention: $\quad 9$ sessions (3 weeks); HIIT: $4 \times 4$ min of running at $90-95 \%$ of max HR/3 min rest; LSD: continuous running at $70-75 \%$ of max HR for $60-80$ $\min$ 
TABLE 4 | Continued
Measures:
Salivary cortisol and immunoglobin-A (slgA); $\mathrm{VO}_{2}$ Peak and Performance
Outcome:
$\mathrm{HIIT}=$ longer time-to-exhaustion and $\uparrow \mathrm{VO}_{2 \text { peak }}$ compared to LSD, slgA secretion rate was higher on the last day of training, as well as the area under the curve (AUCG) higher on the first and last day of training and follow-up compared to the LSD. The AUCG for cortisol remained unaffected on the first and last day of training but increased on the follow-up day with both, HIIT and LSD. slgA secretion rate with the HIIT indicates no compromised mucosal immune function

Bartlett et al. (2020)

Objective:

Determine if neutrophil functions could be improved in association with changes in fitness and metabolic parameters in older adults at risk for Type 2 Diabetes Mellitus using 10-weeks of low volume high-intensity interval exercise training (HIIT)

Participants:

Intervention:

Ten older sedentary adults with prediabetes completed 10 weeks of a supervised HIIT program

Measures: $10 \times 60$ s intervals at $80-90 \%$ Heart rate reserve/50-60\% HRR rest

Before and after training, $\mathrm{VO}_{2 p e a k}$, glucose and insulin sensitivity, neutrophil chemotaxis, bacterial phagocytosis, reactive oxygen species (ROS) production, and mitochondrial functions were assessed $\left(\mathrm{VO}_{2 \text { peak }}\right.$ and neutrophil functions were compared to six young (23 \pm 1 years) healthy adults)

Outcome:

Significant $\downarrow$ in fasting glucose and insulin were accompanied by $\uparrow$ glucose control and insulin sensitivity; $\mathrm{VO}_{2} \mathrm{Peak} \uparrow 16 \pm 11 \%$; Following training, chemotaxis phagocytosis and stimulated ROS $\uparrow$ while basal ROS $\downarrow$ similar to levels observed in the young controls; mitochondrial functions $\uparrow$ toward those observed in young controls, $\downarrow$ the deficit of the young controls between

Dorneles et al. (2019)

Objective:

Participants:

Intervention:

Measures:

To verify the effect of 1 week of high-intensity interval training (HIIT) on the peripheral frequency of T helper subsets and monocyte subtypes Seven sedentary obese men

One week of HIIT ( $3 \times /$ week) -10 bouts of $60 \mathrm{~s}\left(85-90 \% \mathrm{HR}_{\max }\right)$ alternated with $75 \mathrm{~s}$ of recovery $\left(50 \% \mathrm{HR}_{\max }\right)$

Blood samples before and $24 \mathrm{~h}$ after the last session for phenotypic analysis of $\mathrm{T}$ cells and monocytes

Outcome:

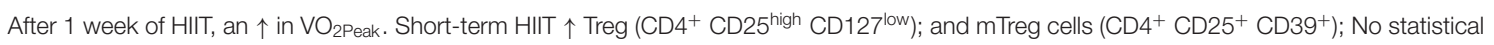
difference was observed in other immune cell phenotypes analyzed

Steckling et al. (2019)

Objective:

Participants:

Intervention:

Measures:

Effects of HIIT on systemic levels of inflammatory and hormonal markers in postmenopausal women with metabolic syndrome (MS)

Fifteen postmenopausal women with MS

Treadmill running $3 \times$ per week, for 12 weeks. $4 \times 4$ min intervals at $90 \% \mathrm{HR}_{\max }$, with 3 min active recovery at $70 \% \mathrm{HR}$ max

Body composition, $\mathrm{VO}_{2 \max }$, serum plasma levels of cytokines (levels of IL-1b, IL-6, IL10, IL-18, TNF-a, interferon-gamma-IFN-c), nitrate and nitrite (NOx) levels, and adiponectin, resistin, leptin, and ghrelin were determined along the intervention

Outcome:

$\mathrm{VO}_{2 \max }$ and anthropometric parameters were $\uparrow$ after HIIT, while $\downarrow$ levels of proinflammatory markers and $\uparrow$ levels of interleukin-10 (IL-10) were also found. Adipokines were also modulated after 12 weeks or training. The mRNA expression of the studied genes was unchanged after HIIT

Kaspar et al. (2016)

Objective:

To compare effect of single-bout endurance (ET) and HIIT on the plasma levels of 4 inflammatory cytokines and C-reactive protein and insulin-like growth factor

Participants:

Seven healthy untrained volunteers

Intervention:

HIIT: 6 sets of $30 \mathrm{~s}$ of all-out supramaximal intensity cycling; ET: 45 min of ergometer cycling at a moderate intensity, which was calculated at $62.5 \%$ of Max HR

Measures: $\quad$ Plasma samples for the interleukins (IL), IL-1 $\quad$, IL-6, and IL-10, monocyte protein-1 (MCP-1), insulin growth factor 1 (IGF-1), and C-reactive protein (CRP)

Outcome:

ET: significant acute and long-term inflammatory response with $\downarrow$ decrease at 30 min after exercise in the IL-6/IL-10 ratio (-20\%) and a $\downarrow$ of MCP-1 (-17.9\%); There were no significant changes in the plasma levels of CRP, IL-1, and IGF-1 from baseline to either 30 min or 2 days after the intervention

exhaustion $-p=0.02 ; \mathrm{VO}_{2 \mathrm{Max}}-p=0.01$ ), induced functional immunoglobin-A adaptations following 4 days of training in recreational adult runners. Furthermore, HIIT promotes similar inflammatory responses after exercise compared to traditional endurance training, suggesting its viability as a training strategy (Kaspar et al., 2016; Bartlett et al., 2017). However, an adequate progression of intensity is suggested to avoid deleterious effects due to high doses of exercise. In the workout model recommended here, despite the fact that it is called high-intensity interval exercise, the effective physiological impact (product of volume vs. intensity) is reasonably small (main workout). Moreover, such proposals mainly focus on recreational trained people. In line with this, several studies have shown significant findings in favor of HIIT protocols when compared to moderateintensity exercise, showing how the immunological system responds to vigorous to high-intensity training with very short duration (Table 4).

\section{FUTURE PERSPECTIVES}

It is reasonable to think that HIIT can also be adjusted to improve physical fitness and health in individuals with low levels of fitness (Gormley et al., 2008), as well as for overweight and obese people, 
according to the trend facing this pandemic (Wewege et al., 2017). First, it is important to understand that the term high intensity should not necessarily be interpreted as a high effort (that would generate limiting condition), since the effort depends on the ratio between intensity and time. In the case of protocols with neuromuscular characteristics, it is possible to establish a suitable threshold for each fitness pattern, mainly controlling the pace with which the movements are performed or the time spent in each stimulus. For cyclic aerobic exercise, the external load, related to the percentage level of $\mathrm{VO}_{2 \mathrm{Max}}$ required by the coach, is in high physical demand, while the internal load, referring to internal perceptions and changes, can modulate a perceived effort to tolerable levels (Foster et al., 2001). Thus, considering the nonprolonged exposure to high-intensity stimuli, we were able to produce significant results for the cardiorespiratory component (Buchheit and Laursen, 2013), as well as important functional adaptations to the immune system (Bartlett et al., 2017), and promote greater adherence to exercise by individuals with a lower level of fitness (Hartman et al., 2019). Therefore, HIIT is expected to be recognized from a safe and effective doseresponse perspective (Taylor et al., 2019) as a potential tool for

\section{REFERENCES}

ACSM (2020). Staying Physically Active During The COVID-19 Pandemic. Available online at: https://www.acsm.org/read-research/newsroom/newsreleases/news-detail/2020/03/16/staying-physically-active-during-covid-19pandemic

Amatriain-Fernandez, S., Gronwald, T., Murillo-Rodriguez, E., Imperatori, C., Solano, A. F., Latini, A., et al. (2020a). Physical exercise potentials against viral diseases like COVID-19 in the elderly. Front. Med. 7:379. doi: $10.3389 /$ fmed.2020.00379

Amatriain-Fernandez, S., Murillo-Rodriguez, E. S., Gronwald, T., Machado, S., and Budde, H. (2020b). Benefits of physical activity and physical exercise in the time of pandemic. Psychol. Trauma 12, S264-S266. doi: 10.1037/tra0000643

Ballesta Garcia, I., Rubio Arias, J. A., Ramos Campo, D. J., Martinez GonzalezMoro, I., and Carrasco Poyatos, M. (2019). High-intensity interval training dosage for heart failure and coronary artery disease cardiac rehabilitation. a systematic review and meta-analysis. Rev. Esp. Cardiol. 72, 233-243. doi: 10.1016/j.rec.2018.02.015

Bartlett, D. B., Shepherd, S. O., Wilson, O. J., Adlan, A. M., Wagenmakers, A. J. M., Shaw, C. S., et al. (2017). Neutrophil and monocyte bactericidal responses to 10 weeks of low-volume high-intensity interval or moderateintensity continuous training in sedentary adults. Oxid. Med. Cell. Longev. 2017:8148742. doi: $10.1155 / 2017 / 8148742$

Bartlett, D. B., Slentz, C. A., Willis, L. H., Hoselton, A., Huebner, J. L., Kraus, V. B., et al. (2020). Rejuvenation of neutrophil functions in association with reduced diabetes risk following ten weeks of low-volume high intensity interval walking in older adults with prediabetes - a pilot study. Front. Immunol. 11:729. doi: 10.3389/fimmu.2020.00729

Bartlett, D. B., Willis, L. H., Slentz, C. A., Hoselton, A., Kelly, L., Huebner, J. L., et al. (2018). Ten weeks of high-intensity interval walk training is associated with reduced disease activity and improved innate immune function in older adults with rheumatoid arthritis: a pilot study. Arthritis. Res. Ther. 20:127. doi: 10.1186/s13075-018-1624-x

Born, D. P., Zinner, C., and Sperlich, B. (2017). The mucosal immune function is not compromised during a period of high-intensity interval training. Is it time to reconsider an old assumption? Front. Physiol. 8:485. doi: 10.3389/fphys.2017.00485

Box, A. G., Feito, Y., Brown, C., Heinrich, K. M., and Petruzzello, S. J. (2019). High Intensity Functional Training (HIFT) and competitions: the improvement of the immune system and consequently for the prevention of respiratory diseases.

\section{AUTHOR CONTRIBUTIONS}

AS participated in the conception of the idea and complete writing of the article, along with SB, CdP, and TM. SM, DT, DM, LC, and CI participated in numerous reviews of this study. CI, TY, and SA participated in the suggestions and the final writing of the article and the adequacy and submission of the study. HB, EM-R, and SM were the main advisers and tutors of all trajectory of studies and designing all phases of the study. All authors contributed to the article and approved the submitted version.

\section{FUNDING}

SM was supported by a grant from Carlos Chagas Foundation for the Research Support in the State of Rio de Janeiro (FAPERJ), Young Scientists from the State of Rio de Janeiro, E26/203.295/2017. how motives differ by length of participation. PLoS ONE 14:e0213812. doi: 10.1371/journal.pone.0213812

Buchheit, M., and Laursen, P. B. (2013). High-intensity interval training, solutions to the programming puzzle: Part I: cardiopulmonary emphasis. Sports Med. 43, 313-338. doi: 10.1007/s40279-013-0029-x

Buckley, S., Knapp, K., Lackie, A., Lewry, C., Horvey, K., Benko, C., et al. (2015). Multimodal high-intensity interval training increases muscle function and metabolic performance in females. Appl. Physiol. Nutr. Metab. 40, 1157-1162. doi: 10.1139/apnm-2015-0238

Chen, P., Mao, L., Nassis, G. P., Harmer, P., Ainsworth, B. E., and Li, F. (2020). Wuhan coronavirus $(2019-\mathrm{nCoV})$ : The need to maintain regular physical activity while taking precautions. J. Sport Health Sci. 9, 103-104. doi: 10.1016/j.jshs.2020.02.001

Dorneles, G. P., da Silva, I., Boeira, M. C., Valentini, D., Fonseca, S. G., Dal Lago, P., et al. (2019). Cardiorespiratory fitness modulates the proportions of monocytes and T helper subsets in lean and obese men. Scand. J. Med, Sci. Sports 29, 1755-1765. doi: 10.1111/sms.13506

Durrer, C., Francois, M., Neudorf, H., and Little, J. P. (2017). Acute high-intensity interval exercise reduces human monocyte Toll-like receptor 2 expression in type 2 diabetes. Am. J. Physiol. Regul. Integr. Comp. Physiol. 312, R529-R538. doi: 10.1152/ajpregu.00348.2016

Emberts, T., Porcari, J., Dobers-Tein, S., Steffen, J., and Foster, C. (2013). Exercise intensity and energy expenditure of a tabata workout. J. Sports. Sci. Med. 12, 612-613.

Foster, C., Florhaug, J. A., Franklin, J., Gottschall, L., Hrovatin, L. A., Parker, S., et al. (2001). A new approach to monitoring exercise training. J. Strength Cond. Res. 15, 109-115. doi: 10.1519/00124278-200102000-00019

Gibala, M. (2009). Molecular responses to high-intensity interval exercise. Appl. Physiol. Nutr. Metab. 34, 428-432. doi: 10.1139/H09-046

Gibala, M. J., Little, J. P., van Essen, M., Wilkin, G. P., Burgomaster, K. A., Safdar, A., et al. (2006). Short-term sprint interval versus traditional endurance training: similar initial adaptations in human skeletal muscle and exercise performance. J. Physiol. 575, 901-911. doi: 10.1113/jphysiol.2006.1 12094

Gormley, S. E., Swain, D. P., High, R., Spina, R. J., Dowling, E. A., Kotipalli, U. S., et al. (2008). Effect of intensity of aerobic training on VO2max. Med. Sci. Sports Exerc. 40, 1336-1343. doi: 10.1249/01.mss.0000321629.41403.46

Hall, G., Laddu, D. R., Phillips, S. A., Lavie, C. J., and Arena, R. (2020). A tale of two pandemics: how will COVID-19 and global trends in physical 
inactivity and sedentary behavior affect one another? Prog. Cardiovasc. Dis. doi: 10.1016/j.pcad.2020.04.005. [Epub ahead of print].

Hartman, M. E., Ekkekakis, P., Dicks, N. D., and Pettitt, R. W. (2019). Dynamics of pleasure-displeasure at the limit of exercise tolerance: conceptualizing the sense of exertional physical fatigue as an affective response. J. Exp. Biol. 222:jeb186585. doi: 10.1242/jeb.186585

Hickson, R. C., Foster, C., Pollock, M. L., Galassi, T. M., and Rich, S. (1985). Reduced training intensities and loss of aerobic power, endurance, and cardiac growth. J. Appl. Physiol. 58, 492-499. doi: 10.1152/jappl.1985.58.2.492

Jamurtas, A. Z., Fatouros, I. G., Deli, C. K., Georgakouli, K., Poulios, A., Draganidis, D., et al. (2018). The effects of acute low-volume hiit and aerobic exercise on leukocyte count and redox status. J. Sports Sci. Med. 17, 501-508.

Kaspar, F., Jelinek, H. F., Perkins, S., Al-Aubaidy, H. A., deJong, B., and Butkowski, E. (2016). Acute-phase inflammatory response to single-bout HIIT and endurance training: a comparative study. Mediat. Inflamm. 2016:5474837. doi: $10.1155 / 2016 / 5474837$

Katzmarzyk, P. T., Powell, K. E., Jakicic, J. M., Troiano, R. P., Piercy, K., and Tennant, B. (2019). Sedentary behavior and health: update from the 2018 physical activity guidelines advisory committee. Med. Sci. Sports Exerc. 51, 1227-1241. doi: 10.1249/MSS.0000000000001935

Khammassi, M., Ouerghi, N., Said, M., Feki, M., Khammassi, Y., Pereira, B., et al. (2020). Continuous moderate-intensity but not high-intensity interval training improves immune function biomarkers in healthy young men. J. Strength Cond. Res. 34, 249-256. doi: 10.1519/JSC.0000000000002737

Kikuchi, N., and Nakazato, K. (2017). Low-load bench press and push-up induce similar muscle hypertrophy and strength gain. J. Exerc. Sci. Fit. 15 37-42. doi: 10.1016/j.jesf.2017.06.003

Lewnard, J. A., and Lo, N. C. (2020). Scientific and ethical basis for socialdistancing interventions against COVID-19. Lancet Infect. Dis. 20, 631-633. doi: 10.1016/S1473-3099(20)30190-0

Nieman, D. C. (2007). Marathon training and immune function. Sports Med. 37, 412-415. doi: 10.2165/00007256-200737040-00036

Paoli, A., Moro, T., Marcolin, G., Neri, M., Bianco, A., Palma, A., et al. (2012). High-Intensity Interval Resistance Training (HIRT) influences resting energy expenditure and respiratory ratio in non-dieting individuals. J. Transl. Med.10:237. doi: 10.1186/1479-5876-10-237

Park, S. E. (2020). Epidemiology, virology, and clinical features of severe acute respiratory syndrome -coronavirus-2 (SARS-CoV-2; Coronavirus Disease-19). Clin. Exp. Pediatr. 63, 119-124. doi: 10.3345/cep.2020.00493
Reljic, D., Lampe, D., Wolf, F., Zopf, Y., Herrmann, H. J., and Fischer, J. (2019) Prevalence and predictors of dropout from high-intensity interval training in sedentary individuals: a meta-analysis. Scand. J. Med. Sci. Sports 29, 1288-1304. doi: $10.1111 /$ sms.13452

Steckling, F. M., Farinha, J. B., Figueiredo, F. D. C., Santos, D. L. D., Bresciani, G., Kretzmann, N. A., et al. (2019). High-intensity interval training improves inflammatory and adipokine profiles in postmenopausal women with metabolic syndrome. Arch. Physiol. Biochem. 125, 85-91. doi: 10.1080/13813455.2018.1437750

Tabata, I., Nishimura, K., Kouzaki, M., Hirai, Y., Ogita, F., Miyachi, M., et al. (1996). Effects of moderate-intensity endurance and highintensity intermittent training on anaerobic capacity and VO2max. Med. Sci. Sports Exerc. 28, 1327-1330. doi: 10.1097/00005768-19961000000018

Taylor, J. L., Holland, D. J., Spathis, J. G., Beetham, K. S., Wisloff, U., Keating, S. E., et al. (2019). Guidelines for the delivery and monitoring of high intensity interval training in clinical populations. Prog. Cardiovasc. Dis. 62, 140-146. doi: 10.1016/j.pcad.2019.01.004

Wewege, M., van den Berg, R., Ward, R. E., and Keech, A. (2017). The effects of high-intensity interval training vs. moderate-intensity continuous training on body composition in overweight and obese adults: a systematic review and meta-analysis. Obes. Rev. 18, 635-646. doi: 10.1111/obr. 12532

WHO (2020). Be Active During COVID-19. Geneva: World Health Organization. Available online at: https://www.who.int/westernpacific/news/q-a-detail/beactive-during-covid-19

Conflict of Interest: The authors declare that the research was conducted in the absence of any commercial or financial relationships that could be construed as a potential conflict of interest.

Copyright (c) 2020 Sá Filho, Miranda, de Paula, Barsanulfo, Teixeira, Monteiro, Cid, Imperatori, Yamamoto, Murillo-Rodriguez, Amatriain Fernández, Budde and Machado. This is an open-access article distributed under the terms of the Creative Commons Attribution License (CC BY). The use, distribution or reproduction in other forums is permitted, provided the original author(s) and the copyright owner(s) are credited and that the original publication in this journal is cited, in accordance with accepted academic practice. No use, distribution or reproduction is permitted which does not comply with these terms. 